\title{
The Eötvös Torsion Balance and its Use in the Field.
}

\author{
By Capt. H. Shaw and E. Lancaster Jones.
}

TH HE problem of locating from the surface mineral deposits in the interior of the earth presents numerous difficulties, and has been considered by many investigators who have employed methods based on the physical properties of these bodies, utilising electrical, magnetic, seismic, and density effects. Speaking generally the useful minerals are of either very high or very low density, so that a method dependent upon the difference in density between the mineral and its surroundings would appear to be suitable for the location of such deposits, especially as these effects are noticeable at a considerable distance.

The attraction due to a heavy body is superimposed upon the normal force of gravity at any point in its vicinity, and it is by observations on this local field by means of a torsion balance that the disturbing mass may be located most readily.

The torsion balance of Michell and Cavendish was redesigned and employed for this purpose in 1888 by Baron Roland von Eötvös, professor of physics at Budapest, who gave to the balance a new construction which is retained essentially in the modern instrument, although numerous improvements have since been introduced.

The essential features of the balance can be seen on reference to Fig. I. A light aluminium beam loaded with platinum weights is suspended by a fine platinum iridium torsion wire. One of the weights is attached directly to one end of the beam, but at the other end the weight is suspended about $60 \mathrm{~cm}$. below the beam by means of a fine wire.

The forces of gravity acting on the two masses are not wholly vertical, but have small horizontal components which give rise to a minute horizontal torque tending to rotate the balance arm. The displacement of the beam relative to its case is observed by means of a telescope and scale fixed to the case, with the aid of a mirror carried by the beam.

When the position of equilibrium of the system has been read, the entire beam and case are rotated through $72^{\circ}$ and the observation repeated. Five observations are thus obtained in one revolution of the balance, and the readings in these positions are sufficient to furnish the information required for the station at which the balance is situated.

The modern type of balance consists of two similar beam systems placed side by side, with the suspended weights at opposite ends, and as with this type only three observations at angles of $120^{\circ}$ are required for a complete determination, a considerable saving of time results. These improved instruments are in some cases fitted with photographic recording arrangements, the balance case being rotated automatically into its new azimuth after the beam has come to rest and the exposure made.

The suspended system requires most careful protection against convection currents and other disturbing influences, and consequently is enclosed in a double or treble-walled brass case of uniform thickness. Temperature and radiation effects are thus reduced considerably, while the additional precaution is taken of working at night, thereby eliminating solar radiation effects and securing a greater constancy of temperature. Under these conditions the instrument is found to give uniform and satisfactory results in the field when protected only by a tent.

In order to secure the necessary degree of sensitivity it is essential that the period of oscillation should be large, and Eötvös has succeeded in obtaining a period of swing of 1500 to 1800 seconds by employing weights of 30 grams and a beam of $40 \mathrm{~cm}$. length, the lower

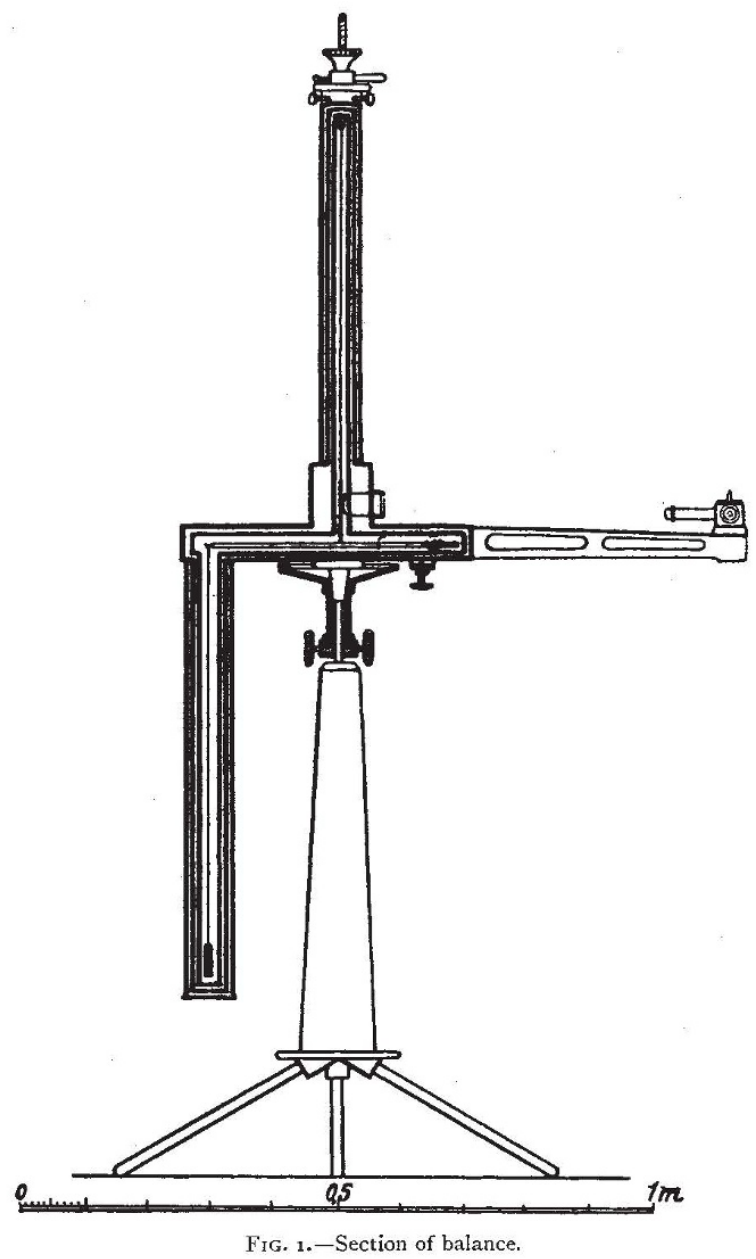

weight being suspended at a depth of about $60 \mathrm{~cm}$. below the beam. The sensitivity of the instrument is partly controlled by the coefficient of torsion of the suspension wire, and by using a platinum wire 0.04 $\mathrm{mm}$. in diameter, alloyed with 20 per cent. iridium, it is possible to measure variations of gravity to within one Io $^{-9}$ C.G.S. unit per centimetre. These wires, which have hitherto proved the most suitable for the purpose, are previously subjected to a special "baking" treatment with the view of eliminating remanent torsion. Similar instruments of smaller dimensions have been constructed and tried by Eötvös, but were subsequently abandoned as being unsatisfactory. Quartz fibres have also been employed in place of the torsion 
wire, but it was found by Eötvös that a quartz fibre which is sufficiently strong to carry the loaded beam is more rigid than the platinum iridium wire and in consequence a smaller deflexion of the beam results. It was partly owing to this fact and partly owing to the exceptional fragility of the quart $z$ fibre that it was not adopted by Eötvös in his field instrument.

The action of the balance and the nature of the quantities measured may be appreciated from the following consideration. Let a system of rectangular co-ordinates $\mathrm{O} x, \mathrm{O} y, \mathrm{O} z$, Fig. 2, have its origin $\mathrm{O}$ at

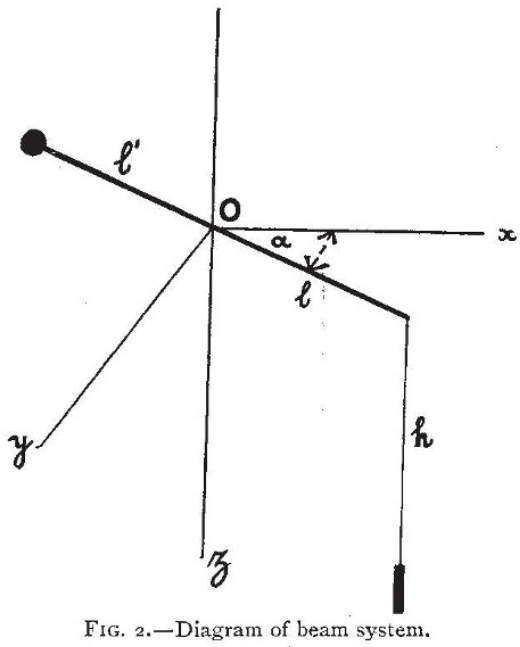

the centre of gravity of the balance beam, the axis $\mathrm{O} z$ directed downwards in the line of resultant gravity at $\mathrm{O}$, and the axes $\mathrm{O} x, \mathrm{O} y$ towards the geographical north and east respectively. It is assumed that a potential function $U$ exists for the gravitational field about $O$ and that, for points not outside the range of swing of the balance beam, we can put

$$
\begin{aligned}
\mathrm{U}=\mathrm{U}_{0}+g_{0} z & +\frac{1}{2} \mathrm{U}_{11} x^{2}+\frac{1}{2} \mathrm{U}_{22} y^{2}+\frac{1}{2} \mathrm{U}_{33^{2}} z^{2} \\
& +\mathrm{U}_{12} x y+\mathrm{U}_{13} x z+\mathrm{U}_{23} y z
\end{aligned}
$$

where $\mathrm{U}_{0}$ is the value of $\mathrm{U}$ at $\mathrm{O}$,

$$
\text { " } g_{0}, \quad, \quad, \text { the resultant force at } \mathrm{O} \text {, }
$$

and $\mathrm{U}_{11}, \mathrm{U}_{12}$, etc. depend only on $\mathrm{O}$, not on $x, y$, and z. Such an assumption is justified whenever gravity is normal, or even if there are irregularities in the field, provided the disturbing masses are fairly distant from the balance.

If the balance beam lies in any position in the plane $\mathrm{O} x y$, making an angle $\alpha$ with the axis $\mathrm{O} x$, its main mass is concentrated at two points of which the co-ordinates are $\left(l^{\prime} \cos (\alpha+\pi), l^{\prime} \sin (\alpha+\pi), 0\right)$ for the upper weight and $(l \cos \alpha, l \sin \alpha, h)$ for the lower. The potential of the whole system will therefore depend on $a$, and will only be a minimum or maximum for a limited number of values of $\alpha$. For all other azimuths the beam will tend to rotate so as to set itself in a position of minimum potential, and will actually rotate until this tendency is balanced by the torsion of the suspension wire. The latter, measured by means of the telescope and scale, affords a means of determining the twisting moment due to the gravitational field at any value $a$, and thus enables us to evaluate the quantities which specify the field and the torque due to it. It is shown in the mathematical theory that these quantities are none other than the magnitudes

$$
\left(\mathrm{U}_{22}-\mathrm{U}_{11}\right), \mathrm{U}_{12}, \mathrm{U}_{13} \text {, and } \mathrm{U}_{23} \text {, }
$$

which are thus determined for every station $\mathrm{O}$.

\section{Application of the Balance.}

The magnitudes $\left(\mathrm{U}_{22}-\mathrm{U}_{11}\right), \mathrm{U}_{12}, \mathrm{U}_{23}, \mathrm{U}_{13}$ determined by the balance are not sufficient to enable us to reproduce the complete gravitational field about $\mathrm{O}$-in other words, to describe its equipotential surfaces--since we require to know both $\mathrm{U}_{11}$ and $\mathrm{U}_{22}$ separately, and also $\mathrm{U}_{33}$ and $g_{0}$. Eötvös, however, has shown that, by means of the four magnitudes determined by his balance, and one or two pendulum measurements, the magnitude of $g$, the force of gravity, can be determined throughout a region where the earth's surface deviates only slightly from the equipotential surface through the base point. The balance is thus of great service to geodesy.

In recent years, however, the balance has been extensively employed for work having a wider appeal than the problems of geodesy. By its capacity to explore the regions below the earth's surface, not by penetrating it but by remaining always on the surface, it has proved itself a valuable ally to the geologist, and its use is superseding much of the costly boring and drilling hitherto necessary in locating mineral deposits. Wherever such deposits differ sufficiently in density from their surroundings, and are sufficiently extensive to cause appreciable irregularities in the gravitational field at the surface above them, the balance not only registers their existence, but also helps to determine their density, shape, extent, and depth below the surface, so that, in favourable circumstances, a single boring may suffice to settle any remaining doubts regarding the nature and size of the deposit. In this work of exploring subterranean disturbing masses, the same four quantities $\left(\mathrm{U}_{22}-\mathrm{U}_{11}\right)$, $\mathrm{U}_{12}, \mathrm{U}_{23}$, and $\mathrm{U}_{13}$ are employed, but the influence of all known disturbing masses, and the normal field due to the size and shape of the earth, must be calculated and eliminated before accurate conclusions regarding the unknown masses can be drawn. This may be a very laborious and complicated process, and may even be impossible in unfavourable regions, e.g., where there are mountain ranges of an irregular character in the vicinity.

In such regions, however, the variations of strata as regards character and shape are frequently sufficiently apparent from surface indications to render the use of the instrument unnecessary, so that the balance is of most use where it is most accurate, namely, in regions presenting a regular and comparatively unbroken surface, but having important irregularities below the surface.

In this work, certain simple combinations of $\left(\mathrm{U}_{22}-\mathrm{U}_{11}\right), \mathrm{U}_{12}, \mathrm{U}_{23}, \mathrm{U}_{13}$, are more useful than the magnitudes themselves. Those mainly used are S, R, $\lambda, \mu$, where

$$
\begin{array}{ll}
\mathrm{S}=\sqrt{\mathrm{U}_{13}{ }^{2}+\mathrm{U}_{23}{ }^{2}}, & \mathrm{R}=-\frac{\mathrm{I}}{g}\left(\mathrm{U}_{22}-\mathrm{U}_{11}\right) \sec 2 \lambda, \\
\tan \mu=\frac{\mathrm{U}_{23}}{\mathrm{U}_{13}}, & \tan 2 \lambda=-\frac{2 \mathrm{U}_{12}}{\mathrm{U}_{22}-\mathrm{U}_{11}} .
\end{array}
$$


The magnitude S represents the " maximum gradient of gravity in the horizontal surface," i.e., the maximum amount by which the vertical force of gravity is increased as we proceed from the origin through unit distance in any direction in the horizontal surface, and is obviously the resultant of $\mathrm{U}_{13}$ and $\mathrm{U}_{23}$, the gradients in the direction $\mathrm{O} x, \mathrm{O} y$ respectively. Also the direc:tion of this maximum gradient is given by $\mu$, the angle which it makes with $\mathrm{Ox}$.

The magnitude $R$ is equal to the difference between the reciprocals of the principal radii of curvature of the level surface at $\mathrm{O}$, and is always positive. Thus, if $\rho_{1}$ is the least radius of curvature at $O$, and $\rho_{2}$ the greatest,

$$
\mathrm{R}=\frac{\mathrm{I}}{\rho_{1}}-\frac{\mathrm{I}}{\rho_{2}},
$$

while $\lambda$ is the angle which the plane of greatest radius of curvature, or least curvature, makes with the plane $\mathrm{O} x z$.

The work of survey consists in finding these values $\mathrm{S}, \mathrm{R}, \lambda, \mu$ at as many stations as possible, correcting them for normal gravity effects and known irregularities, and plotting the final values, representing the maximum gradient $\mathrm{S}$ by an arrow drawn through the station in the direction $\mu$, and proportional in length to the magnitude of $S$, and indicating $R$ by another arrow in the direction $\lambda$. The positions, directions, and lengths of these arrows are then compared with the corresponding arrows given by certain simple mass distributions of which the effects can be calculated, and the probable distribution corresponding to the observed results is deduced.

To illustrate the gravitational effect of a subterranean mass and the variation of the magnitudes measured by the balance from point to point on the earth's surface above such a deposit, we may consider the simple case represented in Fig. 3. Here a horizontal layer of matter, having a density greater by unity than its surroundings, is bounded on the top and bottom by horizontal surfaces at depths 200 and 300 metres below the earth's surface. The layer extends to infinity in the north, east, and west directions, but terminates at the south end in a vertical plane through the east-west line. Let $O$ be a point on the earth's surface on the line where this vertical boundary meets the latter, $x^{\prime} \mathrm{O} x$ the meridian through $\mathrm{O}$, and $\mathrm{O} z$ the downwards vertical meeting the faces of the deposit in $\mathrm{A}$ and $\mathrm{B}$. Consider the force of gravity due to the deposit alone-which is thus to be regarded as having a density unity-at any point $\mathrm{X}$ on $x^{\prime} \mathrm{O} x$. The force at $\mathrm{X}$ will be wholly in the plane $x \mathrm{O} z$, and the corresponding potential surface through $\mathrm{X}$ will be a cylinder having its axis perpendicular to this plane. In these circumstances the magnitudes $\mathrm{U}_{11}, \mathrm{U}_{12}$, etc., specifying

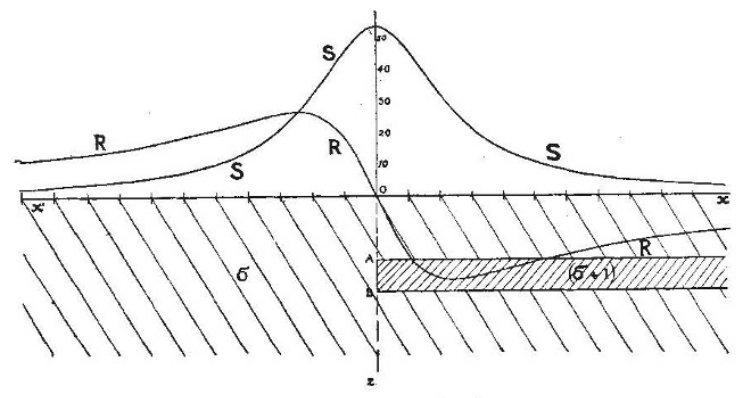

Fig. 3.-Results for a simple case.

the disturbing field due to the deposit, can easily be calculated. Moreover we have

and therefore

$$
\begin{gathered}
\mathrm{U}_{12}=\mathrm{U}_{22}=\mathrm{U}_{23}=\mathrm{O}, \\
\lambda=\mu=\mathrm{O} \text { or } \pi, \\
\mathrm{R}=\mathrm{U}_{11}, \\
\mathrm{~S}=\mathrm{U}_{13} .
\end{gathered}
$$

In Fig. 3 the values of $\mathrm{U}_{\mathbf{1 1}}$ are plotted as ordinates corresponding to the abscissæ $O X$ in the curve RRR, and the values of $U_{13}$ in the curve SSS. It will be noticed that the point $O$, vertically above the edge of the deposit, is strongly marked in each curve by a maximum on one and a zero value on the other. The maximum value of $\mathrm{S}$ has a magnitude $53 \times 10^{-9}$ C.G.S. units, and the maximum of $R$ is $26 \times 10^{-9}$ units. Since values of $R$ and $S$ as low as $I \times 10^{-9}$ unit affect the balance, it is apparent that the instrument would readily show the effects due to such a subterranean deposit, and indicate its extremity.

\section{Science and Industry in Sweden.}

THE exhibition recently opened at Gothenburg to celebrate the tercentenary of the founding of that city by. Gustavus Adolphus, with its display of Swedish manufactures, is an eloquent reminder of the part taken by Sweden in the development of certain industries and also of the debt of the world to Swedish men of science. Though she cannot lay claim to mathematicians of the rank of Leibnitz, Newton, or Euler, or to astronomers equal to Galileo or Herschel, in chemistry and mineralogy Sweden has often led the way, and few countries can boist of names more widely known than those of Bergmann, Scheele, Gadolin, Berzelius, Nilson, Cleve, and Arrhenius.

The rise of science in Sweden is generally traced to Linnæus, but it really had its foundation in the middle of the seventeenth century. Like all the western nations Sweden felt the influence of the clis- coveries of Copernicus, Kepler, and Galileo, and one of the objects the young and eccentric Queen Christina had in view when she invited Descartes to her capital, was to place him at the head of the academy she proposed to establish. The plans of Christina, however, came to nothing, for Descartes died in $165^{\circ}$ and four years later she herself abdicated.

Sweden has a comparatively large territory but a very limited population. Until recent times there were but two seats of learning, Uppsala and Lund. Both are still small cities, the former having about 20,000 inhabitants, the latter some 4000 less. Uppsala is about 40 miles north of Stockholm, while Lund is not far from Malmö in the extreme south. Lund University was founded in 1666 , Uppsala in 1476. It was in Uppsala that Swedish science had its birth, and there it has found its principal home. Johann Gestrin and Magnus Celsius (1621-1679) were among 